\section{Bioinformatics Analysis, Cloning and Expression of Spirometra erinaceieuropaei Fatty Acid-Binding Protein}

\author{
Lin Huang ${ }^{1}$, Ling Mai' ${ }^{2}$ Keyan Zhong ${ }^{1}$ and Xinjun Chen ${ }^{3 *}$ \\ ${ }^{1}$ Experimental Animal Center for Teaching, Hainan Medical University, Haikou 571199, \\ P.R. China. \\ ${ }^{2}$ Department of Pathology, Danzhou People's Hospital, Danzhou 571747, P.R. China. \\ ${ }^{3}$ Laboratory of Pathogenic Biology and Immunology, Hainan Medical University, \\ Haikou 571199, P.R. China.
}

Lin Huang and Ling Mai contributed equally to this work.

\begin{abstract}
A B S T R A C T
To clarify the characteristics of Spirometra erinaceieuropaei fatty acid-binding protein (SeFABP) and obtain its recombinant protein, the basic physio-biochemistry characteristics, signal peptides, antigen epitopes, transmembrane domains, secondary and tertiary structures, multi sequence alignment and molecular evolutionary tree of SeFABP were predicted and analyzed. On this basis, SeFABP was wholegenome synthesized and cloned into prokaryotic expression vector. The recombinant plasmid $\mathrm{pET}$ 30a(+)-SeFABP was constructed, then transformed into E. coli BL21 (DE3) and induced by IPTG. The purified SeFABP was obtained by Ni-IDA resins affinity chromatography, and were finally confirmed by SDS-PAGE and Western blot. The results showed that SeFABP, a stable intracellular protein without signal peptide and transmembrane region, was composed of 130 amino acids (AA). It contained 10 phosphorylation sites and 7 post-translational modification sites. Both methods predicted that SeFABP contained 5 linear epitopes, and were highly coincident, indicating that SeFABP had good immunogenicity. Its secondary structure contains $2 \alpha$-helix $(13.07 \%), 10 \beta$-sheet $(56.92 \%)$ and $30 \%$ random coli. The comparison of multiple FABPs sequences showed that the species were identical and highly conserved at multiple AA sites. Specifically, SeFABP has the highest similarity with T. multiceps and $M$. vogae, close to $37 \%$, but the lowest similarity with $F$. hepatica and $F$. gigatica, close to $22 \%$. Plasmid double enzyme digestion and sequencing showed that the fully synthesized SeFABP fragment was accurately cloned and connected to the expression vector pET-30a(+)-SeFABP. After induction, the recombinant SeFABP was successfully expressed in the form of inclusion body. SDS-PAGE and Western blot showed a 15kD band, indicating that the recombinant was successfully purified. In conclusion, the characteristics of SeFABP and its high-purity recombinant protein were obtained, provides a basis for future research based on SeFABP in vaccine development and drug design.
\end{abstract}

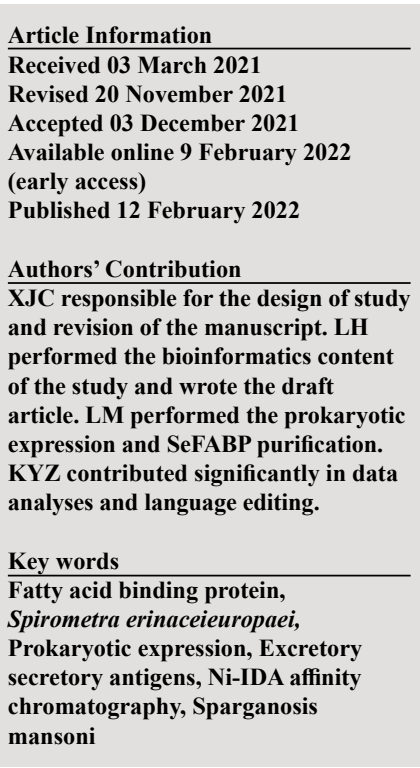

\section{INTRODUCTION}

$\mathrm{F}$ atty acid-binding protein (FABP) is a widely distributed polygenic cytoplasmic protein with low molecular weight and high affinity with fatty acids. FABP accounts for the majority of excretory secretory proteins and is widely distributed in specific parasitic tissues (Alvite and Esteves, 2012). With the function of FABP, parasites oxidize fatty acids transported from the host to meet their own growth and development needs (Gomez-Fuentes et al., 2020). FABPs often show immunogenicity during infection, trigger allergic antibodies, and are related to immune protection,

\footnotetext{
* Corresponding author: chenxj@hainmc.edu.cn 0030-9923/2022/0003-1027 \$ 9.00/0

Copyright 2022 Zoological Society of Pakistan
}

which is closely related to the immune avoidance of parasites (Parks et al., 2021).

In view of the high antigenicity in the life history of parasites, FABPs has been selected as one of the six candidate vaccine antigens for schistosomiasis control (Molehin, 2020). Vaccines made from FABP genes of Schistosoma japonicum and Schistosoma mansoni have been proved to significantly reduce the burden of parasites (McManus, 2021) and show good immune protection (Eyayu et al., 2020). Inspired by Schistosoma vaccine, more FABPs vaccines have been developed (Esteves, 2009), including $F$. hepatica, F. gigantica, E. granulosus, B. malayi (Zhan et al., 2018). In addition, FABPs can also be used as diagnostic antigen. A $14.5 \mathrm{kda}$ FABP of Fasciola hepatica is designed as a sandwich ELISA. The sensitivity and specificity for serological diagnosis of 
Fasciola hepatica are more than 95\% (Allam et al., 2012). Moreover, FABPs are also star candidate molecules for the treatment of chronic inflammatory diseases in parasite 'worm therapy'. Experimental studies clearly show that parasitic FABPs have great anti-inflammatory potential in vitro (Ramos-Benítez et al., 2017) and animal disease models (Bélgamo et al., 2020; Hajizadeh et al., 2021). The above studies all show that parasite FABPs has broad prospects as a biological therapeutic drug.

Spirometra erinaceieuropaei is a parasite of cats and occasionally humans. Its larva sparganosis mansoni can cause sparganosis, which is widely prevalent in Southeast Asian countries, and relevant cases are often reported in China (Zhang et al., 2020). Due to the lack of specific diagnostic methods, misdiagnosis often occurs in clinic. If specific antigen screening can be carried out for the serological diagnosis of sparganosis, the detection rate can be improved, which will be of great help to the clinic (Hu et al., 2014). In previous work at our university, the full-length cDNA plasmid library of Spirometra erinaceieuropaei had been constructed by smart method (Lv et al., 2010), which provides a solid foundation for cloning and identifying key parasitic proteins such as FABP that can be used in vaccines or molecular drugs.

In this study, we first performed bioinformatics analysis of SeFABP by using various bioinformatics tools; then, the whole gene was synthesized and connected with the cloning vector to induce the expression of SeFABP, and then purified by Ni-IDA affinity chromatography. Finally, the quality and specificity of the recombinant SeFABP were confirmed by western blot and SDS-PAGE. This study aims to lay a foundation for evaluating the potential of SeFABP as a diagnostic antigen and its application in 'worm therapy' in clinical treatment.

\section{MATERIALS AND METHODS}

\section{Bioinformatics analysis of SeFABP}

The mRNA of SeFABP was obtained from GenBank (Accession No. JQ919795.1). ProtParam was used to predict the protein molecular weight, isoelectric point, amino acid (AA) sequencing, extinction coefficient, estimated half-life and instability index. ProtScale was used to predict the hydrophilicity/hydrophobicity. TMHMM and Signal $P$ were used to predict transmembrane domains and signal peptides. TargetP and EukmPLoc were used to predict the subcellular location. Netpho3.0 and PROSITE were used to predict phosphorylation sites and post-translational modification sites, respectively. The linear B-cell epitopes of SeFABP were predicted by BepiPred (V2.0) and ABCpred. The secondary structure was predicted by PSIPRED (V4.0). The tertiary structure was predicted by phyre2 based on homology model. Two methods in SAVES, Verify3D and PROCHECK, were used to evaluate the quality of 3D model. The FABPs sequences of several related parasites were compared with cluster Omega, and the comparison results were further processed by Jalview, and the molecular evolution was constructed by adjacency method in Mega X.

\section{Construction of SeFABP prokaryotic expression vector}

SeFABP was whole-genome synthesized (Merrybio, Nanjing). Two digestion sites NdeI and HindIII were designed upstream and downstream, and His-tag was introduced. Then, the SeFABP gene fragment was inserted into the expression vector pET-30a(+) and transformed into competent DH-5 $\alpha$ (Tiangen, Beijing). The recombinant expression vector pET-30a(+)-SeFABP was constructed. After transformation, it was spread on LB plate (kanamycin $50 \mu \mathrm{g} / \mathrm{mL}$ ) and cultured at $37^{\circ} \mathrm{C}$ for 14 $\mathrm{h}$. The positive colonies were selected and cultured with LB liquid medium at $37{ }^{\circ} \mathrm{C}$ for $16 \mathrm{~h}$. After that, bacteria were collected and plasmids were extracted with Plasmid Extraction Kit (Tiangen, Beijing), and was digested by restriction enzymes ApaI and HindIII (New England Biolabs, USA), then Sanger sequencing (MERRYBIO, Nanjing) was performed.

\section{Induced expression of SeFABP protein}

The recombinant vector was transformed into BL21 (DE3) cells (Tiangen, Beijing) and evenly coated on LB plate (containing $50 \mu \mathrm{g} / \mathrm{mL}$ Kan), incubated overnight at $37{ }^{\circ} \mathrm{C}$. A single clone was selected from the plate and inoculated into $4 \mathrm{~mL}$ LB medium $(50 \mu \mathrm{g} / \mathrm{mL}$ Kan $)$. When OD600 reached to $0.8,0.5 \mathrm{mM}$ IPTG (sigma, USA) was added to the culture and maintained at $37^{\circ} \mathrm{C}$ for $16 \mathrm{~h}$. Then was centrifuged at $12000 \mathrm{rpm}$ for $5 \mathrm{~min}$, removed the supernatant, resuspended with PBS, and sonicated with $\mathrm{pH}$ 8.0, $20 \mathrm{mM}$ Tris (BBI, Canada), $300 \mathrm{mM} \mathrm{NaCl}$, $20 \mathrm{mM}$ imidazole (containing 1\% Triton X-100, BBI, Canada), $1 \mathrm{mM}$ DTT (SANGON, Shanghai) and $1 \mathrm{mM}$ PMSF (Thermo Scientific, USA). Then the supernatant and sediment were analyzed by SDS-PAGE.

\section{Purification of SeFABP and verification of protein specificity}

SeFABP appeared in the form of inclusion body, thus it was first cleaned with $20 \mathrm{~mm}$ Tris with $\mathrm{pH} 8.0,300 \mathrm{mM}$ $\mathrm{NaCl}$ with $1 \%$ Triton X-100, 2 mM EDTA (BBI, Canada) and $5 \mathrm{mM}$ DTT. Then, were lysed with $20 \mathrm{mM}$ Tris (pH 8.0 ), $300 \mathrm{mM} \mathrm{NaCl}, 8 \mathrm{M}$ urea (sigma Aldrich, USA) and $20 \mathrm{mM}$ imidazole buffer, and the Ni-IDA (novagen, USA) column was balanced. Finally, the target protein was eluted with different concentrations of imidazole buffer $(50 \mathrm{mM}$, 
$300 \mathrm{mM}$ ), and each eluted fraction was collected for SDSPAGE analysis. After purification by Ni-IDA affinity chromatography, pure SeFABP was collected and added to the dialysis bag and dialyzed into the following buffer (BBI, Canada), $(1 \times$ Denaturation was performed in PBS at $\mathrm{pH}$ 8.0, 4 mm GSH, $0.4 \mathrm{~mm}$ GSSG, $0.4 \mathrm{~m} \mathrm{L-arginine,} 1$ $\mathrm{M}$ urea, $5 \%$ glycerol) at $4{ }^{\circ} \mathrm{C}$. SeFABP ended at $1 \times$ PBS ( $\mathrm{pH} 8.0$ ), dialysed in 5\% Glycorol for about 6-8 h. Then the supernatant was filtered through a $0.22 \mu \mathrm{m}$ filter and incubated with SDS-PAGE and anti-his labeled antibody respectively for Western blot.

\section{RESULTS}

Physical and chemical properties of SeFABP

The total length of SeFABP mRNA sequence is 446 bp. The largest ORF starts from $1 \mathrm{bp}$ and ends with $393 \mathrm{bp}$, encoding a protein of $130 \mathrm{AA}$. The sequence is as follows:

MEAFCGSWKLKDSGDLEPIANRLGVSIPLKDIAELLNSIMRISPVGDGYSMQISNGAMTYEMKYKLGEEFDHASLDGRPLKTKVTLEGKTLKQVDKGDKLMTMESVVEGNTLTMTARLEELVCVRRYTRI.

Its molecular formula: $\mathrm{C}_{633} \mathrm{H}_{1041} \mathrm{~N}_{171} \mathrm{O}_{197} \mathrm{~S}_{10}$, molecular weight is $14.52 \mathrm{kDa}$ and $\mathrm{pI}$ is 5.88 . The estimated half-life in mammals is $30 \mathrm{~h}$, which is longer than that in E. coli $(10$ h) and in yeast $(20 \mathrm{~h})$. Its molar absorption coefficient $(\varepsilon)$ at $280 \mathrm{~nm}$ is $11585 \mathrm{~mol}^{-1} \mathrm{~cm}^{-1}$ when it is assumed that all a pair of Cys residues form cystine. When in $0.1 \%$ ABS (1 $\mathrm{g} / \mathrm{L}), \varepsilon$ is $0.789 \mathrm{~mol}^{-1} \mathrm{~cm}^{-1}$. The aliphatic index was 88.46 and the hydrophobic average index (GRAVY) was -0.283 , indicating that SeFABP is a hydrophobic protein. SeFABP has multiple hydrophilic and hydrophobic regions (Fig. 1A). However, the hydrophilic region is dominant. It is mainly distributed in 8-19, 44-53 and 56-102 AAs.

The $\mathrm{C}, \mathrm{S}$ and $\mathrm{Y}$ values of the predicted sequence processed by Signalp 4.1 showed that all were less than 0.5 , indicating that SeFABP had no signal peptide. In addition, the transmembrane structure was not predicted through TMHMM, both indicating that it is a non-transmembrane protein (Fig. 1B, C). The expected possibility of SeFABP found through TargetP as a signal peptide was determined to be 0.0001 and 0.0003 as a mitochondrial protein. However, the highest probability of localization in other parts of the cell was 0.9995 , indicating that SeFABP is an intracellular protein, which was also verified by EukmPLoc 2.0.

Posttranslational modification sites of SeFABP and prediction of linear $B$ cell epitopes

SeFABP contained 5 serine phosphorylation sites, which were located at $7,13,43,74$ and 105 AA residues respectively (Fig. 2), 4 threonine phosphorylation were located at 85, 90, 113 and 128 AA residues, a tyrosine phosphorylation was located at AA residue 49, respectively.

In addition, 7 potential post-translational modification sites were found: 3 phosphorylation of protein kinase $\mathrm{C}$ (7-9 Swk, 90-92 Tik, 115-117 TaR), a phosphorylation of casein kinase II (105-108 SvvE), an N-myristoylation (109-114: GNtITM), a cAMP and cGMP dependent protein kinase phosphorylation (125-128), and a microbodies C-terminal targeting signal (128-130 TRI). The predicted epitope sequence is summarized in Table I. Two different methods were used and all had predicted the 5 epitopes, suggesting that SeFABP has strong antigenicity. Both methods predicted 4 main overlapping regions $(9-18,27-$ 30, 68-80 and 95-104 AAs), showing that the results were highly reliable.

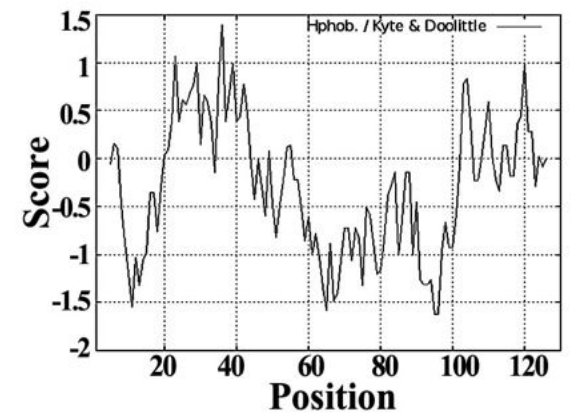

A

Fig. 1. Analysis of basic physical and chemical characteristics of SeFABP.

A, Protscale predicts the hydrophilicity/hydrophobicity of SeFABP. B, The signal peptide of SeFABP analyzed by SignalP. C, SeFABP transmembrane domain predicted by TMHMM. 


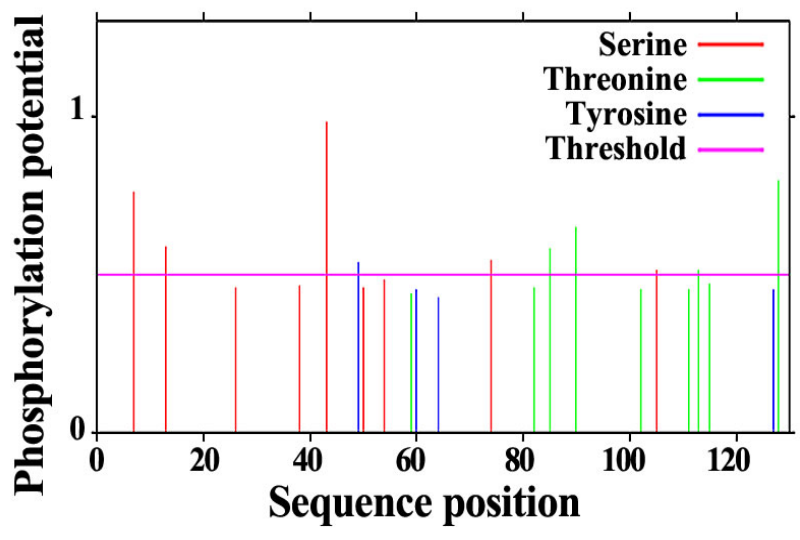

Fig. 2. The phosphorylation site of SeFABP predicted by NetPho3.1

Table I. The epitopes of SeFABP analyzed by BepiPred and ABCpred.

\begin{tabular}{lllll}
\hline Method & Start & End & Peptide & Length \\
\hline BepiPred & 9 & 18 & KLKDSGDLEP & 10 \\
BepiPred & 27 & 30 & IPLK & 4 \\
BepiPred & 32 & 34 & IAE & 3 \\
BepiPred & 68 & 80 & EEFDHASLDGRPL & 13 \\
BepiPred & 95 & 104 & DKGDKLMTM & 10 \\
ABCpred & 9 & 18 & KLKDSGDLEP & 10 \\
ABCpred & 24 & 32 & GVSIPLKDI & 9 \\
ABCpred & 68 & 83 & EEFDHASLDGRPLKTK & 16 \\
ABCpred & 96 & 111 & KGDKLMTMESVVEGNT & 16 \\
ABCpred & 111 & 120 & TLTMTARLEE & 10 \\
\hline
\end{tabular}

Secondary structure and prediction of three-dimensional structure of SeFABP

In the secondary structure, $17 \mathrm{AAs}$ located in $\alpha$-helix region accounts for about $13.07 \%$ of the total AA (Fig. 3A), while $37 \mathrm{AAs}$ located in the random-coli region account for $30 \%$. The proportion of $\beta$-sheets was the highest $(56.92 \%)$, where 74 AAs were located. Its secondary structure showed that SeFABP with high proportion of random coli may bind to antibody more easily. According to homology modeling, the final predicted three-dimensional structure consists of two short $\alpha$-helix and ten long $\beta$-sheet, which is basically consistent with the prediction of the above secondary structure. It is also similar to the structure of most reported parasite FABP proteins (Fig. 3B).

Two methods, verify3D and ERRAT in SAVES, were selected to evaluate the quality of the model. The 3D-1D score was obtained by Verify 3D according to the model quality (Fig. 4A), more than $80 \%$ residues was greater than 0.2, indicating that the predicted SeFABP was a qualified model. The results of PROCHECK showed the Laplacian diagram of the model structure (Fig. 4B), 95\% of the residues fall within the red allowable region and the positive yellow additional allowable region, and only three residues fall into other regions. Overall, the evaluation results showed that the generated model was structurally reliable and of good quality.

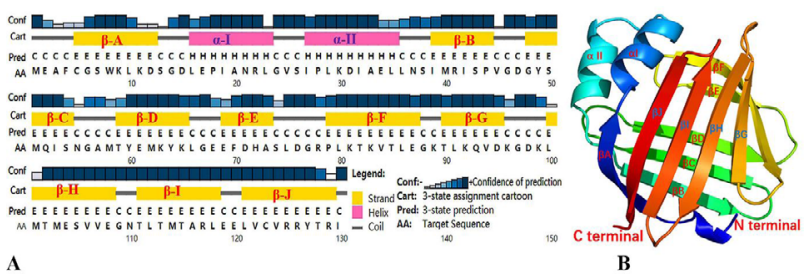

Fig. 3. Prediction of secondary and tertiary structures of SeFABP. (A) Secondary structure predicted by PSIPRED. (B) 3D structure predicted by Phyre2. Both results showed two short $\alpha$-helixs, marked $\alpha$ - I, $\alpha$ - II and ten long $\beta$-sheets, marked as $\beta-\mathrm{A}, \beta-\mathrm{B}$... $\beta$ - J.

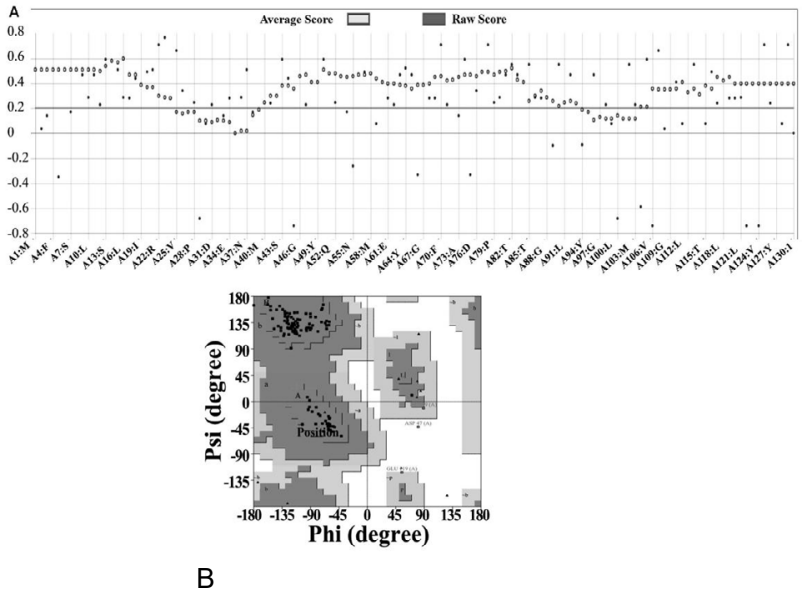

Fig. 4. Quality evaluation of three-dimensional model of SeFABP. (A) The score of Verify3d. (B) Ramachandran diagram analysis of PROCHECK.

Multiple sequence alignment and the phylogenetic analysis of SeFABP

AA sequences of FABP of 10 different parasitic species were retrieved from UniProt, including: Taenia multiceps (ADQ55926.1), Clonorchis sinensis (AAN04089.1), Schistosoma japonicum (AAA64426.1), Echinococcus granulosus (AAK00579.1), Fasciola gigantica (ADK74337.1), Echinococcus multilocularis (CUT99756.1), Hymenolepis microstoma (CDS27711.1), Mesocestoides vogae (ABO93625.3), Fasciola hepatica (THD20935.1) and Schistosoma mansoni (AAA63516.1). 
SeFABP had multiple conservative sites that were the same as other species (Fig. 5), the AAs 1, 4, 6, 8 and 9 of these species were the same, showing a strong conservatism. The sequence similarity between SeFABP and $F$. hepatica was the lowest (about 22\%); even compared with $M$. vogae that had the highest similarity, that was only about $37 \%$ indicating SeFABP still had more variation sites than other reported FABPs. The N-J evolutionary tree in Figure 6 showed that SeFABP had the highest homology with $H$. microstoma and the lowest homology with $F$. hepatica. Trematoda and Taenia formed two different branches in phylogeny. While the homology between SeFABP and other Taenia is not too high, indicating that there are still some evolutionary differences among FABP of different Taenia.

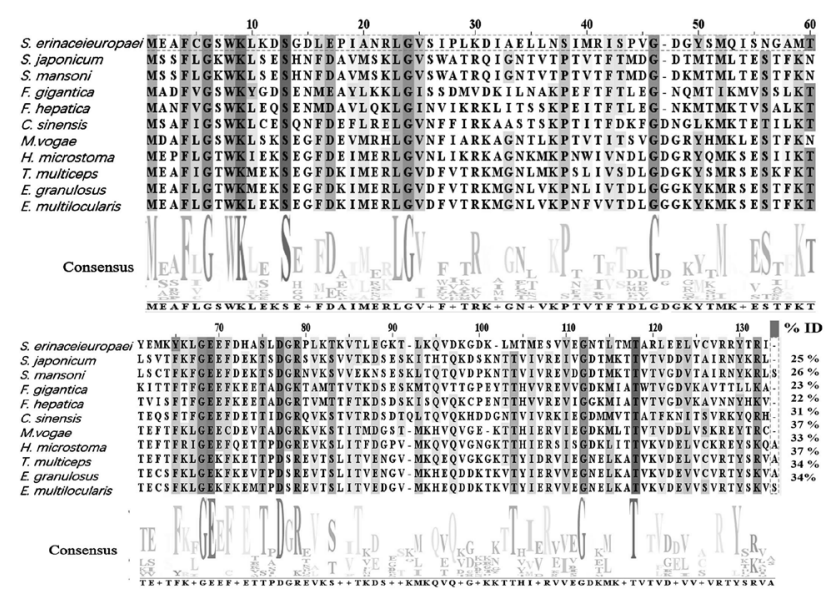

Fig. 5. Multiple sequence alignment of SeFABP and other related parasitic species.

Conservative sites are listed in different colors. All sequences were aligned by Clustal Omega and further edited by Jalview. The letters on consensus represent the same AA residues in these sequences. The percentage similarity of each sequence to SeFABP is displayed in the column marked "ID\%".

\section{Induced expression of recombinant SeFABP}

The construction of pET-30a(+)-SeFABP is shown in Figure 7A. The accuracy of the recombinant expression plasmid was determined by double digestion through ApaI and HindIII, and two fragments, about 4216 and 1451 bp, respectively, were produced (Fig. 7B). The sum of the lengths of the two fragments was consistent with the expected plasmid fragment size.

In addition, the restriction enzyme digestion products of the recombinant plasmid were further sequenced, the results showed that the target gene in the recombinant plasmid pET-30a(+)-SeFABP was 100\% consistent with the corresponding sequence in GenBank, which proved that the target gene had been correctly inserted into pET-30a vector. A clear $15 \mathrm{kDa}$ band was shown in lanes 1 and lane 3 in Figure 7C. The results showed that the recombinant SeFABP was fully expressed under the stimulation of $0.5 \mathrm{~mm}$ IPTG and the induction temperature of $37^{\circ} \mathrm{C}$ for $16 \mathrm{~h}$. However, no recombinant protein was detected in the supernatant (Lane 2), indicating that sefabp was expressed in the form of inclusion body.

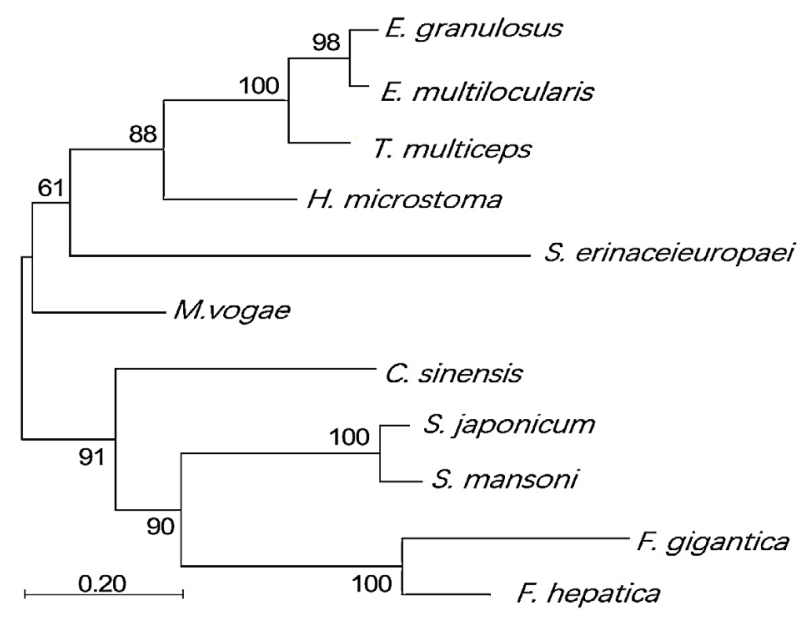

Fig. 6. Phylogenetic analysis of SeFABP with other tapeworms and trematodes.

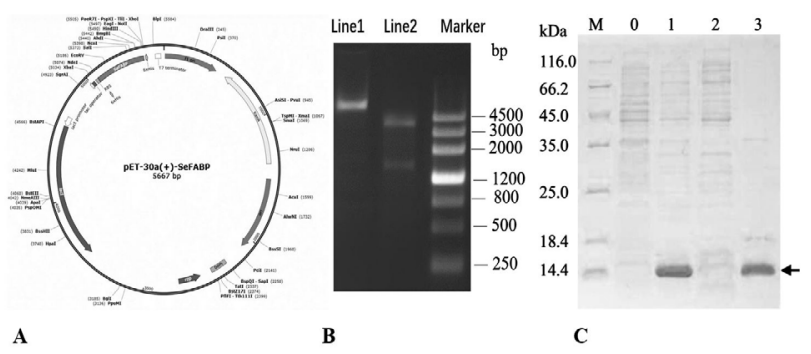

Fig. 7. The expression and identification of SeFABP. A, Schematic diagram of expression vector pET-30a(+) -SeFABP. B, The confirmation of the accuracy of vector. Lane 1: plasmid DNA. Lane 2: digested by ApaI and HindIII. C, The expression of SeFABP identified by SDSPAGE. Lane M, SDS-PAGE protein marker. Lane 0 , control (no IPTG). Lane 1 , induced at $0.5 \mathrm{~mm}$ IPTG at $37^{\circ} \mathrm{C}$ for $16 \mathrm{~h}$. Lane 2, supernatant after bacterial fragmentation. Lane 3, sediment after bacterial fragmentation

\section{Purification of recombinant SeFABP}

Ni-IDA affinity chromatography was used to purify the inclusion body. The purified products of different concentrations of imidazole were collected. It can be seen from Figure 8A that the target protein exists in channels 3, 4 and 5 in the eluent with 50 and $300 \mathrm{mmol} / \mathrm{L}$ imidazole 
concentration, but the band is the clearest and most abundant at $300 \mathrm{mmol} / \mathrm{L}$. Therefore, it can be inferred that the purification effect is the best at the concentration of $300 \mathrm{mmol} / \mathrm{L}$ imidazole. After taking out 4-5 high-purity lanes, dialysis and renaturation of the product were carried out, and then SDS-PAGE electrophoresis was carried out again. A specific band of about $15 \mathrm{kD}$ was detected, which was consistent with the predicted size of SeFABP. Western blot analysis using the specific antibody anti-His also showed a clear band whose size was consistent with the location of SDS-PAGE. These two validation results show that SeFABP is in the form of monomer and has high purity (Fig. 8B). Therefore, it can be used for a series of follow-up studies.

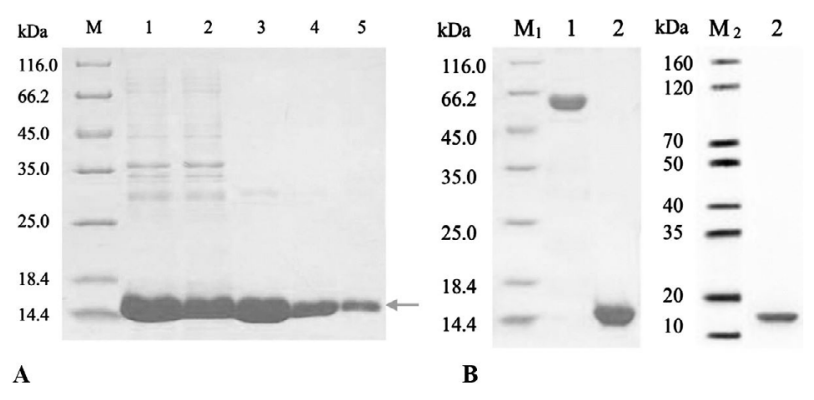

Fig. 8. Purification and specificity verification of recombinant SeFABP. A, The purification results of SeFABP in inclusion bodies were analyzed by SDS-PAGE. Lane M, SDS-PAGE protein marker. Lane 1, supernatant after inclusion body dissolution and centrifugation. Lane 2, effluent after incubation of supernatant with Ni IDA. Lane3, elution component of $50 \mathrm{~mm}$ imidazole. Lane4-5, elution component of $300 \mathrm{~mm}$ imidazole. B, The quality and specificity of FABP were confirmed by SDS-PAGE and Western blot. Lane1, BSA (1.50 $\mu \mathrm{g})$; Lane 2, SeFABP; M1, SDS-PAGE marker. M2, Western blot marker.

\section{DISCUSSION}

FABP plays an important role in the absorption, transport and metabolic regulation of long-chain fatty acids and is an essential key protein for parasites (Pórfido et al., 2020). With the discovery of FABPs of various parasitic species, studies have confirmed that these FABPs occupy part of excretory secretory antigens and show immunogenicity in parasitic infection, thus inducing a protective mechanism inseparable from parasitic immune escape (Corral-Ruiz and Sánchez-Torres, 2020). Therefore, FABP is considered as a potential and promising anti parasitic vaccine (Dalton et al., 2004). For example, the first identified FABP protein of the parasite, Schistosoma mansoni FABP (Tendler et al., 1996), has been developed as a vaccine, showing good efficacy against Schistosoma mansoni (Qokoyi et al., 2021).

It is a necessary step in epitope based vaccine design to predict the immunogenicity linear region on the protein surface. In this study, three epitope sequences predicted by the two methods are very similar and partially overlap with the location of E. granulosus FABP epitope designed as vaccine (Tendler et al., 1996). They are 9-14 (KMEKSE), 68-73 (GEKFKE) and 95-101 (EQDDKTK). Although the sequences of overlapping positions are not exactly the same, it still suggests that the three sequences on SeFABP can be used as antigen epitopes to design vaccines to prevent Spirometra erinaceieuropaei infection, but they still need to be further verified in animal models.

The structure and function of protein are closely related. The secondary structure is the premise of the highlevel structure analysis, because it is the bridge between AA sequence and its three-dimensional conformation. The most abundant secondary structure is $\beta$-sheet, accounting for $56 \%$, while random coli is reaching half of the $\beta$-sheet, accounts for about $30 \%$ of the total AA, four of the five epitopes are located in random coli, indicating that this structure is very easy to bind to antibodies. SeFABP has a similar tertiary structure with most reported parasite FABP, consisting of 10 parallel $\beta$-sheet and two $\alpha$-helix (Kim et al., 2012). Clamshell $\beta$-sheet is a typical feature of intracellular lipid-binding protein (Esteves and Paulino, 2013), and together with $\alpha$-helix, can establish an inner cavity to form the ligand binding site of hydrophobic molecules (Esteves and Ehrlich, 2006), so that the ligands can be better captured and released.

It is well known that phosphorylation is an important and universal post-translational modification of proteins. The process of protein phosphorylation can greatly affect the normal physiological state of parasites, such as the invasion and escape of parasites, as well as their own signal transduction pathway. For example, tyrosine phosphorylation of FABP can regulate the binding of fatty acids (Sha et al., 1993). Bioinformatics predicts that SeFABP has multiple phosphorylation sites, which is consistent with FABPs predicted on Fasciola species (Morphew et al., 2016). These results suggest that SeFABP may regulate protein function through these sites and affect protein activity. Since the phosphorylation of FABP protein is relatively rare in mammalian species (Storch and Corsico, 2008), the predicted phosphorylation sites of SeFABP also indicate differences in the regulation of FA transport between hosts and parasites. In future, in-depth study on the phosphorylation modification of SeFABP is very important to understand the role of FABP in the growth and development of Spirometra erinaceieuropaei, and can provide a basis for screening candidate vaccines or new drug targets of parasitic diseases. 
Traditional methods of obtaining parasitic proteins require the collection of naturally infected intermediate hosts or experimentally infected animals (Chung and Yang, 2008). Therefore, this operation is complex, timeconsuming and laborious, and the protein content and quality of each preparation are different, which greatly limits its further application. The above problems can be solved by cloning and recombinant expression technology (Knox et al., 2001), which is also the most mainstream tool in parasite research. Therefore, on the basis of bioinformatics analysis, we completely synthesized SeFABP gene and constructed prokaryotic expression vector pET-30a(+)-SeFABP. After induction with $0.5 \mathrm{~mm}$ IPTG, the recombinant SeFABP was successfully fused with 6X-histag in the bacterial system, but expressed in the form of inclusion body, which is also consistent with most research reports. The exact mechanism of its formation is not completely clear, but when foreign genes are effectively expressed in E. coli expression system, they often form inclusion bodies (Singhvi et al., 2020). It is speculated that the possible reason is that when the recombinant protein is expressed at a high level, there is not enough time for the normal structure to fold in the cell, so as to form amorphous amorphous protein aggregates. The purified SeFABP was finally obtained by Ni-IDA affinity chromatography, and then double confirmed by SDS-PAGE and Western blot. The results show that there is a clear band at about $15 \mathrm{kD}$, which is consistent with the expected molecular weight of SeFABP plus the size of histidine label, and also consistent with the reported conclusion that the molecular weight of most parasite FABP is usually between $13-15 \mathrm{k}$ da (Nie et al., 2013). This shows that we have obtained recombinant SeFABP with high specificity and sensitivity through molecular biology technology, which provides a reliable and stable protein source for follow-up research.

\section{CONCLUSION}

In this study, the biological characteristics of SeFABP were fully investigated, and its purified recombinant was obtained. It lays a theoretical and research foundation for the functional research of SeFABP.

\section{ACKNOWLEDGEMENT}

This study was supported by the Natural Science Foundation of Hainan Province, China (No. 819 QN235) and the Research Incubation Fund Project on Hainan medical University (No. HYPY2020029).

\section{Statement of conflict of interest}

The authors have declared no conflict of interests.

\section{REFERENCES}

Allam, G., Bauomy, I.R., Hemyeda, Z.M. and Sakran, T.F., 2012. Evaluation of a $14.5 \mathrm{kDa}-$ Fasciola gigantica fatty acid-binding protein as a diagnostic antigen for human fascioliasis. Parasitol. Res., 110: 1863-1871. https://doi.org/10.1007/s00436-0112711-y

Alvite, G. and Esteves, A., 2012. Lipid binding proteins from parasitic platyhelminthes. Front. Physiol., 3: 363-370. https://doi.org/10.3389/fphys.2012.00363

Bélgamo, J.A., Alberca, L.N., Pórfido, J.L., Romero, F.N.C., Rodriguez, S., Talevi, A.B., Córsico, B. and Franchini, G.R., 2020. Application of target repositioning and in silico screening to exploit fatty acid binding proteins FABPs from Echinococcus multilocularis as possible drug targets. J. comp. Aided Mol. Des., 34: 1275-1288. https://doi. org/10.1007/s10822-020-00352-8

Chung, Y.B. and Yang, H.J., 2008. Partial purification and characterization of a cysteine protease inhibitor from the plerocercoid of Spirometra erinacei. Korean J. Parasitol., 46: 183-186. https://doi. org/10.3347/kjp.2008.46.3.183

Corral-Ruiz, G.M. and Sánchez-Torres, L.E., 2020. Fasciola hepatica-derived molecules as potential immunomodulators. Acta Trop., 210: 105548. https://doi.org/10.1016/j.actatropica.2020.105548

Dalton, J.P., Skelly, P and Halton, D.W., 2004. Role of the tegument and gut in nutrient uptake by parasitic platyhelminths. Can. J. Zool., 82: 211-232. https:// doi.org/10.1139/z03-213

Esteves, A. and Ehrlich, R., 2006. Invertebrate intracellular fatty acid binding proteins. Comp. Biochem. Physiol. C Toxicol. Pharmacol., 142: 262274. https://doi.org/10.1016/j.cbpc.2005.11.006

Esteves, A. and Paulino, M., 2013. In silico studies of Echinococcus granulosus FABPs. J. Biomol. Struct. Dyn., 31: 224-239. https://doi.org/10.1080 /07391102.2012.698246

Esteves, A., 2009. Plathylemimth fatty acid binding proteins as candidate vaccines. In: Fatty acid binding proteins (ed. A. Esteves). Transworld Research Network Press, Kerala. pp. 69-87.

Eyayu, T., Zeleke, A.J. and Worku, L., 2020. Current status and future prospects of protein vaccine candidates against Schistosoma mansoni infection. Parasit. Epidemiol. Contr., 11: e00176. https://doi. org/10.1016/j.parepi.2020.e00176

Gomez-Fuentes, S., Morales-Ruiz, V.D., LópezRecinos, D.A., Guevara-Salinas, A., Arce-Sillas, 
J.F., Rodríguez, C., Parada-Colin and AdalidPeralta, L., 2019. Biological role of excretorysecretory proteins in endemic parasites of Latin America and the Caribbean. J. Helminthol., 94: e53. https://doi.org/10.1017/S0022149X19000312

Hajizadeh, M., Saboor-Yaraghi, A.A., Meamar, A.R., Khoshmirsafa, M., Razmjou, E., Sadeghipour, A., Bagheri, Y., Sadeghi, F., Jalallou N., Kazemi, M.H., Salari, A.A. and Falak, R., 2021. The fatty acidbinding protein FABP decreases the clinical signs and modulates immune responses in a mouse model of experimental autoimmune encephalomyelitis EAE. Int. Immunopharmacol., 96: 107756. https:// doi.org/10.1016/j.intimp.2021.107756

Hu, D.D., Cui, J., Xiao, D., Wang, L., Liu, L.N., Liu, R.D., Zhang, J.Z. and Wang, Z.Q., 2014. Identification of early diagnostic antigens from Spirometra erinaceieuropaei sparganum soluble proteins using immunoproteomics. Southeast Asian J. Trop. Med. Publ. Hlth., 45: 576-583.

Kim, S.H., Bae, Y.A., Yang, H.J., Shin, J.H., DiazCamacho, S.P., Nawa, Y., Kang, I. and Kong, Y., 2012. Structural and binding properties of two paralogous fatty acid-binding proteins of Taenia solium metacestode. PLoS Negl. Trop. Dis., 6: e1868. https://doi.org/10.1371/journal. pntd. 0001868

Knox, D.P., Redmond, D.L., Skuce, P.J. and Newlands, G.F., 2001. The contribution of molecular biology to the development of vaccines against nematode and trematode parasites of domestic ruminants. Vet Parasitol., 101: 311-335. https://doi.org/10.1016/ S0304-4017(01)00558-1

Lv, G., Lu, Y.J., Fan, Z.G., Shi, D.Z., Gan, X.F. and Zhong, S.F., 2010. Construction and identification of a full-length cDNA library from Spirometra erinaceieuropaei. Chinese J. Parasitol. Parasit. Dis., 28: 393-394.

McManus, D.P., 2021. The search for a schistosomiasis vaccine: Australia's contribution. Vaccines, 9: 872886. https://doi.org/10.3390/vaccines 9080872

Molehin, A.J., 2020. Schistosomiasis vaccine development: Update on human clinical trials. J. biomed. Sci., 27: 1-7. https://doi.org/10.1186/ s12929-020-0621-y

Morphew, R.M., Wilkinson, T.J., Mackintosh, N., Jahndel, V., Paterson, S., McVeigh, P., Abbas Abidi, S.M., Saifullah, K., Raman, M., Ravikumar, G., LaCourse, J., Maule, A. and Brophy, P.M., 2016. Exploring and expanding the fatty-acidbinding protein superfamily in Fasciola species. J. Proteome Res., 15: 3308-3321. https://doi. org/10.1021/acs.jproteome.6b00331

Nie, H.M., Xie, Y., Fu, Y., Yang, Y.D., Gu, X.B., Wang, S.X., Peng, X., Lai, W.M. and Yang, G.Y., 2013. Cloning and characterization of the fatty acidbinding protein gene from the protoscolex of Taenia multiceps. Parasitol. Res., 112: 1833-1839. https://doi.org/10.1007/s00436-013-3328-0

Parks, S.C., Nguyen, S., Nasrolahi, S., Bhat, C., Juncaj, D., Lu, D., Ramaswamy, R., Dhillon, H., Fujiwara, H., Buchman, A., Akbari, O.S., Yamanaka, N., Boulanger, M.J. and Dillman, A.R., 2021. Parasitic nematode fatty acid-and retinol-binding proteins compromise host immunity by interfering with host lipid signaling pathways. PLoS Pathog., 17: e1010027. https://doi.org/10.1371/journal. ppat. 1010027

Pórfido, J.L., Herz, M., Kiss, F., Kamenetzky, L., Brehm, K., Rosenzvit, M.C., Córsico, B. and Franchini, G.R., 2020. Fatty acid-binding proteins in Echinococcus spp.: The family has grown. Parasitol. Res., 119: 1401-1408. https://doi. org/10.1007/s00436-020-06631-5

Qokoyi, N.K., Masamba, P. and Kappo, A.P., 2021. Proteins as targets in anti-schistosomal drug discovery and vaccine development. Vaccines, 9: 762-791. https://doi.org/10.3390/vaccines 9070762

Ramos-Benítez, M.J., Ruiz-Jiménez, C., Aguayo, V. and Espino, A.M., 2017. Recombinant Fasciola hepatica fatty acid-binding protein suppresses tolllike receptor stimulation in response to multiple bacterial ligands. Sci. Rep., 7: 5455. https://doi. org/10.1038/s41598-017-05735-w

Sha, R., Kane, C.D., Xu, Z., Banaszak, L.J. and Bernlohr, D.A., 1993. Modulation of ligand binding affinity of the adipocyte lipid-binding protein by selective mutation. Analysis in vitro and in situ. J. biol. Chem., 268: 7885-7892. https://doi.org/10.1016/ S0021-9258(18)53040-4

Singhvi, P., Saneja, A., Srichandan, S. and Panda, A.K., 2020. Bacterial inclusion bodies: A treasure trove of bioactive proteins. Trends Biotechnol., 38: 474486. https://doi.org/10.1016/j.tibtech.2019.12.011

Storch, J. and Corsico, B., 2008. The emerging functions and mechanisms of mammalian fatty acid binding proteins. Annu. Rev. Nutr., 28: 73-95. https://doi. org/10.1146/annurev.nutr.27.061406.093710

Tendler, M., Brito, C.A., Vilar, M.M., Serra-Freire, N., Diogo, C.M., Almeida, M.S., Delbem, A.C., Da Silva, J.F., Savino, W., Garratt, R.C., Katz, N. and Simpson, A.S., 1996. A Schistosoma mansoni fatty acid-binding protein, Sm14, is the potential basis of a dual-purpose anti-helminth vaccine. Proc. 
natl. Acad. Sci. U. S. A., 93: 269-273. https://doi. org/10.1073/pnas.93.1.269

Zhan, B., Arumugam, S., Kennedy, M.W., Tricoche, N., Lian, L.Y., Asojo, O.A., Bennuru, S., Bottazzi, M.E., Hotez, P.J. and Lustigman, S., 2018. Ligand binding properties of two Brugia malayi fatty acid and retinol FAR binding proteins and their vaccine efficacies against challenge infection in gerbils. PLoS Negl. Trop. Dis., 12: e0006772. https://doi. org/10.1371/journal.pntd.0006772

Zhang, X., Hong, X., Liu, P., Jiang, S.C., Zhao, C.X., Sun, Z.Q. and Cui, J., 2020. Large-scale survey of a neglected agent of sparganosis Spirometra erinaceieuropaei (Cestoda: Diphyllobothriidae) in wild frogs in China. PLoS Negl. Trop. Dis., 14: e0008019. https://doi.org/10.1371/journal. pntd.0008019 